\title{
Influence of C16:0 and long-chain saturated fatty acids on normal variation of bovine milk fat triacylglycerol structure
}

\author{
D. A. Tzompa-Sosa, ${ }^{*}$ G. A. van Aken, † A. C. M. van Hooijdonk, ${ }^{*}$ and H. J. F. van Valenberg ${ }^{* 1}$ \\ *Dairy Science and Technology Group, Food Quality and Design, Wageningen University, PO Box 17, 6700 AA, Wageningen, the Netherlands \\ †NIZO Food Research, PO Box 2, 6710 BA, Ede, the Netherlands
}

\section{ABSTRACT}

Fatty acids (FA) are nonrandomly distributed within milk fat triacylglycerols (TAG). Moreover, the structure of milk fat TAG differs with feeding regimens. So far, nothing is known about the variation of milk fat TAG structure among individual cows. A deep understanding of the normal variation of TAG structures and the relationships between milk fat FA profile and its TAG structure could help to better control functional and compositional differences between milk fats from various sources and to increase the knowledge on milk fat synthesis. The focus of the present study was to determine the regiospecific TAG structure of individual samples of winter milk fat from Dutch Holstein-Friesian cows with a wide variation of FA profiles and with 2 diacylglycerol acyltransferase 1 (DGAT1) genotypes: DGAT1 K232A genotype AA and DGAT1 K232A genotype KK. From an initial set of 1,918 individual milk fat samples, 24 were selected. The selected samples had a wide range of FA composition and had either DGAT1 K232A genotype AA or KK. The structure analysis was done with a regiospecific approach. This analysis is based on the acyl degradation of TAG by a Grignard reagent and further isolation of $s n-2$ monoacylglycerols by thinlayer chromatography. An intra- and interpositional approach was used to study the structural variation. With the intrapositional approach, the amount of an FA at the secondary $(s n-2)$ and primary $(s n-1,3)$ positions was related to its total amount in the TAG. With the interpositional approach, the proportion of C8:0, C10:0, C14:1 cis-9, C16:1 cis-9, and C18:1 cis-9 at sn-2 was positively correlated with the amount of C16:0 in the triacylglycerol; in contrast, saturated C14:0, C16:0, and long-chain saturated FA (C14:0-C18:0) were negatively correlated. These observations suggest that the amount of long-chain saturated FA in TAG influences the positioning of other FA in the TAG. With an interpositional approach, the DGAT1 polymorphism had

Received January 13, 2014.

Accepted March 28, 2014.

${ }^{1}$ Corresponding author: hein.vanvalenberg@wur.nl a significant effect on the proportional positioning of C16:0 at sn-2. These results provide a new direction to controlling functional and compositional differences between milk fats.

Key words: DGAT1, triacylglycerol structure, regiospecific analysis, glycerol-3-phosphate acyltransferase

\section{INTRODUCTION}

Bovine milk fat is mainly composed (98\%) of triacylglycerols (TAG), which are formed of $3 \mathrm{FA}$ esterified to a glycerol backbone (Jensen, 2002). The most abundant FA in bovine milk fat are C16:0 (32.6 wt\%, on average), C18:1 cis-9 (18.0 wt\%, on average), C14:0 (11.6 wt\%, on average), and C18:0 (8.7 wt\%, on average; Heck et al., 2012). The abundance of these FA is related to the cow diet and to the cow genetic characteristics (Palmquist et al., 1993; Schennink et al., 2007). A diet rich in fresh grasses, for instance, will increase the amount of unsaturated FA (UFA), such as C18:1, n-3, and n-6 FA in milk fat; in contrast, a diet based on maize silage or rich in hay will increase the amount of SFA in milk fat (Palmquist, 2006; van Valenberg et al., 2013). Genetic differences also play a role in FA abundance. For instance, the diacylglycerol acyltransferase 1 (DGAT1) K232A polymorphism has been associated with an increase in C16:0 and a decrease in C14:0, unsaturated C18:1, and CLA concentrations (Schennink et al., 2007). Moreover, C16:0 and C18:1 cis-9 concentrations are negatively correlated and their concentrations are affected by the fat content of milk fat (Stoop et al., 2008).

Fatty acids within bovine milk fat TAG are nonrandomly distributed (Parodi, 1979). A regiospecific approach can be used to study the distribution of FA in the TAG, which allows a differentiation of FA esterified in the primary $(\boldsymbol{s n} \mathbf{- 1}, \mathbf{3})$ and secondary position $(\boldsymbol{s n}$-2). In milk fat TAG, short-chain FA and C18:0 are predominantly located at $s n-1,3$, whereas C14:0 is predominantly located at $s n-2$. Furthermore, C16:0 and C18:1 cis-9 are abundant at $s n-1,3$ and $s n-2$ (Angers et al., 1998; Blasi et al., 2008). The distribution of FA within the TAG can be affected by the nutrition of dairy cows (Morrison and Hawke, 1977; Parodi, 1979; 
Christie and Clapperton, 1982,), which can vary with the season. However, no differences in distribution of FA on TAG in milk fat has been reported between seasons (Parodi, 1979), which could be a consequence of pooling the milk from different farms. Hence, in pooled milk, the seasonality is lost due to a variety of breeds, feeding regimens, and lactation stages. A modification in the TAG structure suggests that the distribution of FA could be triggered by differences in blood-derived FA or by changes in the activity of enzymes related to fat synthesis that respond to availability of FA for TAG synthesis.

The positioning of the FA in the TAG affects the metabolic fate of ingested FA. Saturated FA at the secondary position are absorbed more efficiently than SFA at the primary positions (Kallio et al., 2006) because FA in the secondary position will be absorbed as 2-monoacylglycerol (2-MAG) and this leads to better emulsification properties during digestion (Mattson et al., 1979). The positioning of FA at the secondary position becomes relevant for newborn babies. It has been reported that infants fed formulas in which $\mathrm{C} 16: 0$ is mainly in the primary position present a lower absorption of this FA than when C16:0 is placed at the secondary position (Tomarelli et al., 1968; López-López et al., 2001).

A deep understanding of the normal variation of TAG structures and the relationship between milk fat FA profile and its TAG structure could help to better control functional and compositional differences between milk fats from various sources. In this study, we determined the TAG structure of individual samples of winter milk fat from 24 Dutch Holstein-Friesian cows: 13 with the DGAT1 K232A genotype AA and 11 with the DGAT1 K232A genotype KK. Our aim was to determine if differences in milk fat TAG structure exist among individual cows and to determine if milk fat FA profile is related to the structure of milk fat TAG.

\section{MATERIALS AND METHODS}

\section{Sample Selection of Individual Milk Fat}

The samples used in this study were part of the Dutch Milk Genomics Initiative (Wageningen, the Netherlands) where morning milk fat samples were collected from 1,918 first-lactation Holstein-Friesian cows on 398 commercial herds in the Netherlands (Schennink et al., 2007; Heck et al., 2012). We studied milk fat from the months February and March, which corresponds to the winter season in the Netherlands. The typical winter diet in the Netherlands consists of $31 \%$ concentrate, $44 \%$ grass silage, and $25 \%$ corn silage (Heck et al.,
2009). We ensured a wide range of FA composition by randomly selecting milk fat samples from individual cows with a fat content within the range of 2.85 to $5.76 \%$ and with either DGAT1 K232A genotype AA or KK. Genotyping of the DGAT1 K232A polymorphism was performed with a TaqMan allelic discrimination assay (Applied Biosystems Inc., Foster city, CA) and was performed as part of a previous study (Schennink et al., 2007). In total, 24 cows were selected: 13 with DGAT1 K232A genotype AA and 11 with DGAT1 K232A genotype KK. To avoid confounding with herd effects in our study, all the 24 selected milk fat samples belonged to different commercial herds.

\section{Fat Extraction of Milk Fat}

To extract the fat fraction from milk, we warmed 100 $\mathrm{mL}$ of milk in a water bath at $28^{\circ} \mathrm{C}$. Then, $5 \mathrm{~mL}$ of $\mathrm{HCl}$ (4 M) was added to the milk and shaken for $45 \mathrm{~min}$ until the fat was clearly separated. Next, the fat phase was washed with cold water. Finally, the fat phase was poured into a centrifuge tube and warmed in an oven at $70^{\circ} \mathrm{C}$ for $60 \mathrm{~min}$ and then centrifuged at $1,643 \times g$ for $10 \mathrm{~min}$ at $20^{\circ} \mathrm{C}$. A clear fat phase was present at the top of the centrifuge tube. This fat was used for regiospecific structure analysis.

\section{Regiospecific Structure Analysis of Milk Fat}

The regiospecific distribution of TAG was determined by a chemical degradation with a Grignard reagent and further isolation of 2-MAG by preparative thin-layer chromatography (TLC; Christie and Moore, 1969). The 2-MAG obtained after Grignard degradation have been proven useful for structure analysis of natural TAG (Becker et al., 1993; Angers et al., 1998; Turon et al., 2003). These acyl moieties allow direct determination of the composition of the native TAG. In this study, ethyl magnesium bromide was used to degrade TAG from milk fat. The degradation degree of TAG was of $62 \%$. After degradation, $s n-2$ MAG were isolated by preparative TLC and their FA composition was determined by capillary gas chromatography with flame ionization detector. The FA composition of $s n-1,3$ was calculated from the composition of $s n-2 \mathrm{MAG}$ and TAG. The TAG degradation was done as follows: $50 \mathrm{mg}$ of anhydrous milk fat was weighted in an oven-dried 10-mL vial hermetically closed with a Teflon cap. Then, $2 \mathrm{~mL}$ of dry diethyl ether was added to dissolve the fat. The fat was allowed to react for $30 \mathrm{~s}$ with $200 \mu \mathrm{L}$ of $3 M$ ethylmagnesium bromide in dry ether [Chemical Abstracts Service (CAS) no. 925-90-6; Sigma-Aldrich Chemie BV, Zwijndrecht, the Netherlands] while being 
stirred; the reaction was stopped with $50 \mu \mathrm{L}$ of glacial acetic acid followed by $1.5 \mathrm{~mL}$ of $10 \%$ boric acid solution. The acyl moieties were extracted 3 times with $2 \mathrm{~mL}$ of diethyl ether saturated with boric acid and subsequently washed 3 times with $2 \%$ sodium bicarbonate, water, and brine (sodium chloride-saturated aqueous solution). The water phase from the mixture was removed using a separator funnel. Then, the solvent phase was dried under nitrogen. To separate the acyl moieties, the residue was dissolved in $100 \mu \mathrm{L}$ of diethyl ether and applied on a silica gel plate $(20 \times$ $20 \mathrm{~cm}, 500-\mu \mathrm{m}$ thickness; Analtech Inc., Newark, DE) previously sprayed with $5 \%$ boric acid in methanol. The plates were developed using chloroform/acetone $(85: 15, \mathrm{vol} / \mathrm{vol}) 1 \%$ acetic acid solution (Turon et al., 2002). Two references for MAG were used: 2-oleoylglycerol and 1-oleoyl-rac-glycerol (Sigma-Aldrich Chemie BV). Iodine vapor was used to stain the acyl moieties. In the TLC plate, $s n-2$ MAG (rate of flow $=0.37$ ) ran ahead of $s n-1,3 \mathrm{MAG}$ (rate of flow $=0.29$ ). The band corresponding to $s n-2$ MAG was scraped from the silica plates and transferred to $10-\mathrm{mL}$ test tubes vials. The glycerides were extracted from the silica gel 2 times with diethyl ether aided by sonication (10 min). Precipitation of the silica was achieved by centrifuging $1,000 \times g$ for $10 \mathrm{~min}$ at $20^{\circ} \mathrm{C}$. The glycerides $(4-5$ $\mathrm{mg})$ corresponding to the $s n-2$ fraction were dissolved in diethyl ether and transferred to a $900-\mu \mathrm{L}$ vial and the solvent was evaporated under a stream of nitrogen.

The FA composition of the isolated $s n-2 \mathrm{MAG}$ and of the unreacted fat was analyzed at the Dutch Milk Controlling Institute Qlip Laboratories (Zutphen, the Netherlands) in accordance with the ISO/IDF (2002) international standard. For both samples, approximately $5 \mathrm{mg}$ was methylated and analyzed for FA composition with gas chromatography with flame ionization detector with a WCOT fused silica column [CP WAX $52 \mathrm{CB}, 30 \mathrm{~m} \times 0.25$ i.d., film thickness of the coating of the stationary phase (Df) $=0.25 \mu \mathrm{m}$; Agilent Technologies, Santa Clara, CA]. This column allows the separation of the 12 most abundant FA.

\section{Calculations for Intra- and Interpositional Approaches}

Intrapositional Approach. With this approach, the amount of an FA reported is relative to all FA at that position. Therefore, the sum of the proportions of all FA present at each position will sum to $100 \%$; with this approach, the total amount of an FA in the TAG is not considered.

The FA profile of $s n-2$ MAG, recovered after Grignard degradation, and the FA profile of the unreacted fat were obtained in grams per $100 \mathrm{~g}$ and were trans- formed to mole percentage. Both profiles were used to calculate the FA profile at $s n-1,3$ by using the formula position $1,3_{\mathrm{FA} i}=\frac{3 \times \mathrm{TAG}_{\mathrm{FA} i}-\text { position } 2_{\mathrm{FA} i}}{2} \quad$ (Christie, 2003), where position 2 is the amount of an FA at $s n-2$ and $\mathrm{TAG}_{\mathrm{FA} i}$ is the amount of the same FA in the unreacted TAG and $i$ is one of the FA in milk fat.

Interpositional Approach. With this approach, the distribution of $1 \mathrm{FA}$ over the 3 positions is determined. The FA profiles obtained in the intrapositional approach were used to calculate the proportion of an FA placed at $s n-2$ and $s n-1,3$. The formulas used were

$$
\begin{aligned}
\% s n-2_{\mathrm{FA} i} & =\frac{\text { position } 2_{\mathrm{FA} i}}{\text { position } 2_{\mathrm{FA} i}+\left(\text { position } 1,3_{\mathrm{FA} i} \times 2\right)} \times 100 \\
\% s n-1,3_{\mathrm{FA} i} & =\frac{\text { position } 1,3_{\mathrm{FA} i} \times 2}{\text { position } 2_{\mathrm{FA} i}+\left(\text { position } 1,3_{\mathrm{FA} i} \times 2\right)} \times 100,
\end{aligned}
$$

where position 2 is the amount of a FA at $s n-2$ (in mol\%) and position 1,3 is the amount of the same FA at $s n-1,3$ (in mol\%) and $i$ is one of the FA in milk fat.

\section{Statistical Analysis}

Analyses were performed using IBM SPSS statistics software (version 21; IBM Corp., Armonk, NY). Concentrations and proportions of FA in the TAG and at $s n-2$ and $s n-1,3$ were subjected to bivariate Pearson correlations. The effects of DGAT1, fat content, FA in the TAG, and their interactions on FA at $s n$ - 2 were estimated using the following model:

$$
\mathrm{y}_{\mathrm{ij}}=\mu+D G A T 1_{\mathrm{i}}+\beta_{1} \mathrm{~F}_{\mathrm{ij}}+\beta_{\mathrm{i} 2}\left(D G A T 1_{\mathrm{i}} \times \mathrm{F}_{\mathrm{ij}}\right)+\mathrm{e}_{\mathrm{ij}},
$$

where $\mathrm{y}_{\mathrm{ij}}$ is the dependent variable, $\mu$ is the overall mean, DGAT1 is a fixed effect of DGAT1 genotype i (AA or $\mathrm{KK}$ ), $\beta_{1}$ is the regression coefficient relative to $\mathrm{F}_{\mathrm{ij}} ; \mathrm{F}_{\mathrm{ij}}$ is the covariable describing the effect of the concentration of an FA in the TAG or fat content, $\beta_{\mathrm{i} 2}$ are the regression coefficients relative to the concentration of an FA in the TAG or fat content within the DGAT1 genotypes; $\left(D G A T 1_{\mathrm{i}} \times \mathrm{F}_{\mathrm{ij}}\right)$ describes the interaction between estimated effects of DGAT1 K232A (AA or KK genotypes) and the concentration of an FA in in the TAG or fat content; and $\mathrm{e}_{\mathrm{ij}}$ is the residual effects, which are assumed to be normally distributed. A significance level of $P<0.05$ was used throughout the study.

\section{RESULTS}

Fatty acids in milk fat TAG are nonrandomly distributed (Parodi, 1979). The distribution of FA within the 
TAG molecule has been previously reported with an intra- and interpositional approach (Blasi et al., 2008). Whereas the intrapositional approach helps to identify the most abundant FA at one position, the interpositional approach helps to understand the distribution of each FA over the positions in the TAG. In the current study, we report and discuss our results based on both approaches (Table 1).

\section{Intrapositional Approach}

In this study, we found that the most abundant FA at sn-2 were C14:0, C16:0, and C18:1 cis-9; and the most abundant FA at $s n-1,3$ were $\mathrm{C} 4: 0, \mathrm{C} 16: 0$, and $\mathrm{C} 18: 1$ cis-9 (Table 1). Short-chain FA, such as C4:0 to C8:0, were almost absent at $s n-2$. Fatty acids C14:0, C16:0, C18:0, and C18:1 cis-9 showed the highest variability in distribution over $s n-2$ and $s n-1,3$; these FA were also the most abundant FA in the TAG. We found significant positive correlations between the total amount of an FA in the TAG and its distribution over $s n-1,3$ and $s n-2$ (Table 2). Short-, medium-, and long-chain FA, for instance, have a positive correlation between their total amounts in the TAG and their amounts present at $s n-1,3$. The total amount of C12:0 in the TAG is positively correlated with its amount at $s n-2$. Moreover, significant negative correlations were found between the total amount of an FA in the TAG and the amount of other FA at $s n-2$ and $s n-1,3$ (Table 2). For instance, the total amount of C16:0 in the TAG was negatively correlated with the amount of C6:0, C8:0, C10:0, and C18:1 cis-9 at sn-1,3 and with the amount of C14:0 and C18:0 at $s n-2$.

\section{Interpositional Approach}

The interpretation of the variability of TAG structures within a cow population is easier with an interpositional approach because it places all the FA under the same scale $(0-100 \%)$. With the interpositional approach, we found that the preferential positioning of FA in the TAG varies according to the FA carbon length and its unsaturation level. Medium- and long-chain SFA, such as C12:0, C14:0, C16:0, and C18:0, prefer the $s n-2$ position (Figure 1). Meanwhile, short-chain FA and UFA, such as C4:0 to C8:0 and C14:1, C16:1, and C18:1 cis-9, prefer the $s n-1,3$ position (Table 1 ). The interpositional approach did not show significant correlations between the total amount of specific FA in the TAG and their amounts at $s n-2$ or $s n-1,3$, except for C16:0. The proportion of most FA at $s n-2$ significantly correlated with the amount of C16:0 and long-chain SFA (C14-C18) in the TAG (Table 3).

\section{Effect of DGAT1 Polymorphism and C16:0 on TAG Structure}

The interaction of the DGAT1 polymorphism and C16:0 in the TAG had a significant effect on C16:0 at $s n-2$. This effect was significant when measured on a mole percentage basis $(P=0.05)$ as well as when measured as a proportion $(P=0.04$; Figure 2$)$. No further effect was seen for fat content or for the interaction of the DGAT1 polymorphism with other FA.

\section{DISCUSSION}

\section{Intrapositional Approach}

Our results are in accordance with previous studies (Parodi, 1979; Turon et al., 2002; Blasi et al., 2008), except for C18:0. In our study, the amount of C18:0 at $s n-2$ was similar to the values reported by Christie and Clapperton (1982); however, almost double the amount was found by Blasi et al., (2008), Parodi (1979), and Turon et al. (2002). The differences found in C18:0 are not likely to be associated with the method used to analyze structure because Christie and Clapperton (1982) used an enzymatic approach, whereas Turon et al. (2002) used a chemical approach, such as the one used in the current study.

Moreover, we found large variability among individual cows, which made it interesting to test for correlations. These correlations were indeed found between short-, medium-, and long-chain SFA (Table 2). These FA have a positive correlation between their total amounts in the TAG and their amounts present at $s n-1,3$. Our observations on correlations between total amount in the TAG and at $s n-2$ and $s n-1,3$ are similar to results previously reported by Parodi (1983). These correlations indicate the complexity of the interactions between FA during milk fat synthesis. The relationships between the total amount of an FA in the TAG and at $s n-2$ or $s n-1,3$ are an important aspect that should be considered in the interpretation and comparison of different studies on milk fat TAG structure.

\section{Interpositional Approach}

In this study, most FA at $s n-2$ were significantly correlated with the amount of C16:0 and long-chain SFA (C14-C18) in the TAG (Table 3). These correlations were unexpected because no reports exist of an influence of C16:0 over positioning of other FA in the TAG. These correlations give an indication that $\mathrm{C} 16: 0$ and other long-chain SFA play a role in the positioning of several FA in the TAG molecule. 
Table 1. Total FA composition (mean $\pm \mathrm{SD} ; \mathrm{n}=24$ ) and regiospecific distribution of FA from milk fat triacylglycerols (TAG) with an intra- and interpositional approach ${ }^{1}$

\begin{tabular}{|c|c|c|c|c|c|c|c|c|c|c|c|}
\hline \multirow[b]{4}{*}{ tem } & & & & & \multicolumn{4}{|c|}{ Intrapositional (mol\%) } & \multicolumn{3}{|c|}{ Interpositional (mol\%) } \\
\hline & \multicolumn{4}{|c|}{ Triacylglycerol (mol\%) } & \multicolumn{2}{|c|}{$s n-2^{2}$} & \multicolumn{2}{|c|}{$s n-1,3^{3}$} & \multicolumn{2}{|c|}{$s n-2 \%^{4}$} & \multirow{2}{*}{$\frac{s n-1,3 \%^{5}}{\alpha, \alpha^{\prime}}$} \\
\hline & \multicolumn{4}{|c|}{$\alpha, \beta, \alpha^{\prime 1}$} & \multicolumn{2}{|c|}{$\beta$} & \multicolumn{2}{|c|}{$\alpha, \alpha^{\prime}$} & \multicolumn{2}{|c|}{$\beta$} & \\
\hline & Min. ${ }^{6}$ & Max. $^{6}$ & Average $\pm \mathrm{SD}$ & Literature $^{7,8}$ & Average $\pm \mathrm{SD}$ & Literature $^{7,8}$ & Average $\pm \mathrm{SD}$ & Literature ${ }^{7,8}$ & Average $\pm \mathrm{SD}$ & Literature $^{8,9}$ & Average $\pm \mathrm{SD}$ \\
\hline content & 2.9 & 5.8 & $4.32 \pm 0.82$ & & & & & & & & \\
\hline Saturation index ${ }^{10}$ & 2.4 & 3.9 & $3.31 \pm 0.43$ & & & & & & & & \\
\hline $\mathrm{C} 4: 0$ & 8.3 & 10.9 & $9.40 \pm 0.78$ & $7.6 ; 6$ & - & $— ; 0.4$ & $14.10 \pm 1.17$ & $11.4 ; 8.8$ & - & $2.2 ; 0$ & 100 \\
\hline C6:0 & 3.9 & 5.2 & $4.45 \pm 0.32$ & $4.2 ; 2.9$ & $0.2 \pm 0.06$ & $0.4 ; 0.9$ & $6.58 \pm 0.51$ & $6.1 ; 3.9$ & $1.48 \pm 4.08$ & $10.1 ; 12$ & $98.52 \pm 4.08$ \\
\hline C8:0 & 2 & 2.6 & $2.15 \pm 0.17$ & $2.1 ; 1.7$ & $0.47 \pm 0.75$ & $1.9 ; 0.2$ & $2.99 \pm 0.43$ & $2.2 ; 2.5$ & $7.16 \pm 11.51$ & 4.7; 52 & $92.84 \pm 11.52$ \\
\hline C10:0 & 3.3 & 4.5 & $3.88 \pm 0.32$ & $4.5 ; 3.4$ & $2.59 \pm 1.88$ & $6.9 ; 1.4$ & $4.52 \pm 1.02$ & $3.3 ; 4.4$ & $22.33 \pm 16.14$ & $12.4 ; 51$ & $77.67 \pm 16.14$ \\
\hline $\mathrm{C} 12: 0$ & 3.1 & 7.2 & $4.61 \pm 0.85$ & $4.9 ; 3.9$ & $4.93 \pm 1.77$ & $7.6 ; 4.8$ & $4.46 \pm 1.17$ & $3.6 ; 3.5$ & $35.87 \pm 11.86$ & $39.1 ; 60$ & $64.13 \pm 11.87$ \\
\hline C14:0 & 9.7 & 13.3 & $11.31 \pm 0.90$ & - & $16.73 \pm 3.66$ & $20.3 ; 22.8$ & $8.59 \pm 1.97$ & $9.7 ; 8.3$ & $49.39 \pm 10.6$ & $53.5 ; 63$ & $50.61 \pm 10.59$ \\
\hline C14:1 cis-9 & 0.9 & 1.9 & $1.34 \pm 0.23$ & - & $0.34 \pm 0.5$ & - & $1.85 \pm 0.38$ & - & $8.25 \pm 11.76$ & & $91.75 \pm 11.76$ \\
\hline C15:0 & 0.8 & 1.5 & $1.11 \pm 0.18$ & - & $0.9 \pm 0.58$ & - & $1.21 \pm 0.35$ & - & $27.24 \pm 17.09$ & & $72.76 \pm 17.09$ \\
\hline C16:0 & 22.3 & 32.5 & $27.90 \pm 3.14$ & $32.4 ; 31.6$ & $40.73 \pm 4.45$ & $42.9 ; 44.1$ & $21.48 \pm 4.94$ & $27.1 ; 25.4$ & $49.22 \pm 7.48$ & $42.8 ; 43$ & $50.78 \pm 7.48$ \\
\hline C16:1 cis-9 & 0.8 & 1.9 & $1.24 \pm 0.26$ & $-; 1.8$ & $0.49 \pm 0.58$ & $-; 2.5$ & $1.62 \pm 0.48$ & -1.5 & $13.37 \pm 15.35$ & $42.2 ;-$ & $86.63 \pm 15.32$ \\
\hline $\mathrm{C} 18: 0$ & 4 & 10.1 & $7.41 \pm 1.15$ & $9.7 ; 6.6$ & $11.47 \pm 3.45$ & $6.2 ; 5.3$ & $5.36 \pm 2.15$ & $1.5 ; 7.3$ & $50.24 \pm 19.53$ & $24.2 ; 18$ & $49.76 \pm 19.53$ \\
\hline C18:1 cis-9 & 17.6 & 23.3 & $17.05 \pm 2.81$ & $13.3^{11} ; 19.2$ & $15.11 \pm 2.89$ & $9.6 ; 15.7^{11}$ & $16.34 \pm 4.26$ & $15.1 ; 21^{11}$ & $33.54 \pm 7.25$ & $24.8 ; 24^{11}$ & $66.46 \pm 7.26$ \\
\hline
\end{tabular}

${ }^{1} \alpha$ and $\alpha^{\prime}$ is the primary position of the TAG structure $(s n-1,3) ; \beta$ is the secondary position of the TAG $(s n-2)$.

${ }^{2}$ Isolated fraction obtained after Grignard degradation of milk fat triglycerides.

${ }^{3}$ Calculated from the FA profile from the secondary position $(s n-2)$ and TAG using the formula position $1,3_{\mathrm{FA} i}=\frac{3 \times \mathrm{TAG}_{\mathrm{FA} i}-\mathrm{position} 2_{\mathrm{FA} i}}{2}$.

${ }^{4}$ Calculated from the FA profile of $s n-2$ and primary position $(s n-1,3)$ in mole percentage using the formula $\% s n-2_{\mathrm{FA} i}=\frac{\text { position } 2_{\mathrm{FA} i}}{\text { position } 2_{\mathrm{FA} i}+\left(\operatorname{position~} 1,3_{\mathrm{FA} i} \times 2\right)} \times 100$.

${ }^{5}$ Calculated from the FA profile of $s n-2$ and $s n-1,3$ in mole percentage using the formula $\% s n-1,3_{\mathrm{FA} i}=\frac{\text { position } 1,3_{\mathrm{FA} i} \times 2}{\text { position } 2_{\mathrm{FA} i}+\left(\text { position } 1,3_{\mathrm{FA} i} \times 2\right)} \times 100$.

${ }^{6}$ Min. = minimum; Max. = maximum

${ }^{7}$ Literature data from Turon et al. (2002).

${ }^{8}$ Literature data from Blasi et al. (2008); $s n-1,3$ data from Blasi et al. (2008) were calculated in the current study from the published data.

${ }^{9}$ Literature data from Morrison and Hawke (1977).

${ }^{10}$ Saturation index $=$ total SFA/total unsaturated FA.

${ }^{11}$ Reported as C18:1 total. 
Table 2. Significant correlations between amount (mol\%) of an FA in the triglyceride and the amount (mol\%) at the secondary $(s n-2)$ and primary $(s n-1,3)$ positions

Table 2. Significant correlations between amount (mol\%) of an FA in the triglyceride and the amount (mol\%) at the secondary $(s n-2)$ and primary $(s n-1,3)$ positions

\begin{tabular}{|c|c|c|c|c|c|c|c|c|c|c|c|c|c|}
\hline \multirow[b]{2}{*}{ FA } & \multicolumn{13}{|c|}{ Triacylglycerol (mol\%) } \\
\hline & $\mathrm{C} 4: 0$ & C6:0 & C8:0 & $\mathrm{C} 10: 0$ & $\mathrm{C} 12: 0$ & C14:0 & C14:1 cis-9 & $\mathrm{C} 15: 0$ & C16:0 & $\mathrm{C} 16: 1$ cis-9 & $\mathrm{C} 18: 0$ & C18:1 cis-9 & LCSFA $^{1}$ \\
\hline \multicolumn{14}{|l|}{$\begin{array}{l}s n-2(\mathrm{~mol} \%) \\
\mathrm{C} 4: 0\end{array}$} \\
\hline $\begin{array}{l}\text { C6:0 } \\
\text { C8:0 }\end{array}$ & & & & & & & & & & & & $-0.37 \dagger$ & \\
\hline & & & & & & & & & $\begin{array}{l}0.44^{*} \\
0.41^{*}\end{array}$ & & & & $0.46^{*}$ \\
\hline $\mathrm{C} 12: 0$ & & & & & $0.46^{*}$ & $0.44^{*}$ & & & & & & $0.43^{*}$ & \\
\hline C14:0 & & & $0.47^{*}$ & $0.44^{*}$ & & & & & $-0.38 \dagger$ & & & & $-0.42^{*}$ \\
\hline $\mathrm{C} 14: 1$ cis-9 & & & & & & & & & $0.39 \dagger$ & & & & $0.43^{*}$ \\
\hline C15:0 & & & & & & & & & & & & & \\
\hline C16:0 & & & & & & & & & & $0.36 \dagger$ & & & \\
\hline C16:1 cis-9 & & & & & & & & & $0.47^{*}$ & & & & $0.52^{* *}$ \\
\hline C18:0 & $0.41 \dagger$ & $0.38 \dagger$ & & & & & & $-0.43^{*}$ & $-0.38 \dagger$ & & & & \\
\hline $\begin{array}{l}\text { C18:1 cis-9 } \\
\text { LCSFA }\end{array}$ & & & & & & & $-0.46^{*}$ & & & & $0.49^{*}$ & & \\
\hline \multicolumn{14}{|l|}{$s n-1,3(\mathrm{~mol} \%)$} \\
\hline $\mathrm{C} 4: 0$ & $1^{* *}$ & $0.84^{* *}$ & $0.41^{* *}$ & & & $-0.34 \dagger$ & & $-0.40 \dagger$ & & $-0.40 \dagger$ & & & \\
\hline $\mathrm{C} 6: 0$ & $0.78^{* *}$ & $0.83^{* *}$ & $0.68^{* *}$ & & & & & & $-0.37 \dagger$ & & & & $-0.43^{*}$ \\
\hline $\mathrm{C} 8: 0$ & & & $0.50^{* *}$ & $0.37 \dagger$ & & & & & $-0.54^{* *}$ & & & & $-0.57^{* *}$ \\
\hline C10:0 & & & & $0.40 \dagger$ & & & & & $-0.42^{*}$ & & & & $-0.48^{*}$ \\
\hline C12:0 & & & & $0.37 \dagger$ & $0.74^{* *}$ & $0.54^{* *}$ & & & & & $-0.37 \dagger$ & & \\
\hline C14:0 & & $-0.36 \dagger$ & $-0.51^{*}$ & & & $0.44^{*}$ & & & $0.36 \dagger$ & $-0.38 \dagger$ & & & $0.49^{*}$ \\
\hline C14:1 cis-9 & & & & & & & $0.77^{* *}$ & & & & $-0.44^{*}$ & & \\
\hline C15:0 & & & & & & & & $0.58^{*}$ & & $0.60^{*}$ & $-0.43 \dagger$ & & $0.55^{* *}$ \\
\hline $\mathrm{C} 16: 0$ & & & & & & & & $0.34 \dagger$ & $0.9^{* *}$ & & $-0.36 \dagger$ & $-0.36 \dagger$ & $0.89^{* *}$ \\
\hline C16:1 cis-9 & & & & & & & & $0.46^{*}$ & & $0.80^{* *}$ & & & \\
\hline C18:0 & & & & & $-0.38 \dagger$ & & & & & & $0.63^{* *}$ & & \\
\hline C18:1 cis-9 & & & & & & & & & $-0.68^{* *}$ & & & & $-0.74 * *$ \\
\hline LCSFA & & & & & & & & & & & & & $0.87^{* *}$ \\
\hline \multicolumn{14}{|c|}{${ }^{1}$ LCSFA = long-chain SFA from C14:0 to C18:0. } \\
\hline
\end{tabular}




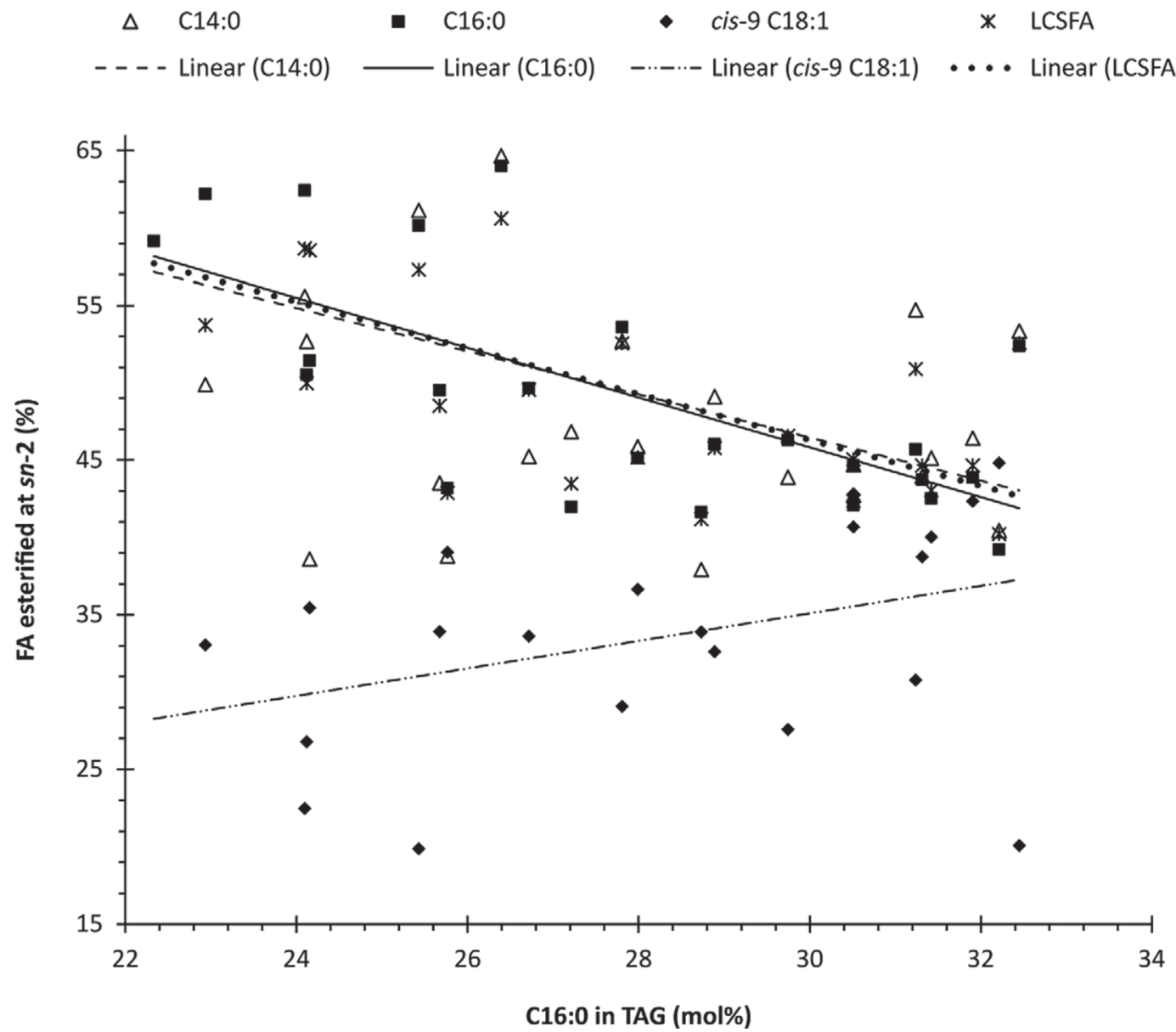

Figure 1. Percentage of C14:0, C16:0, and C18:1 cis-9 esterified at the secondary position (sn-2) and its relationship to the total amount of C16:0 in the triacylglycerol (TAG; mol\%). Percentage C14:0 at $s n-2=88.327-1.396 \times$ C16:0 in TAG $\left(\mathrm{R}^{2}=0.17 ; P=0.04\right)$. Percentage C16:0 at $s n-2=94.134-1.61 \times \mathrm{C} 16: 0$ in TAG $\left(\mathrm{R}^{2}=0.45 ; P<0.0001\right)$. Percentage $\mathrm{C} 18: 1$ cis-9 at sn-2 $=8.367+0.891 \times \mathrm{C} 16: 0$ in TAG $\left(\mathrm{R}^{2}=0.13\right.$; $P=0.1)$. Percentage long-chain SFA (LCSFA) at $s n-2=110.02-2.198 \times \mathrm{C} 16: 0$ in TAG $\left(\mathrm{R}^{2}=0.37 ; P=0.002\right)$.

Previous studies have reported the TAG structure with an interpositional approach (Morrison and Hawke, 1977; Blasi et al., 2008). The interpositional approach was used in those studies to compare milk fat structures from different species or bovine milk fat TAG structures from cows with different diets. The proportions of an FA at $s n$-2 show large variation among studies and might look contradictory. However, as shown in the current study, the proportions of an FA at $s n-2$ depend on the original amount of C16:0 and long-chain SFA (C14-C18) in the TAG. This remark should always be considered when interpreting the results.

\section{Role of C16:0 in Milk Fat TAG Structure}

The most abundant FA in milk fat is $\mathrm{C} 16$ :0; this FA varies greatly between diets (Jensen, 2002). Our results show that the proportion of other $\mathrm{FA}$ at $s n-2$ was correlated with the total amount of C16:0 in the TAG. This arrangement may be related to FA prefer- ence of enzymes involved in milk fat TAG synthesis. So far, only glycerol-3-phospate acyltransferase (GPAT), which esterifies the FA at $s n-1$, was shown to have a preference for SFA at the expense of UFA (Monroy et al., 1973; Bremer et al., 1976; Coleman and Lee, 2004). Glycerol-3-phospate acyltransferase has 2 isoforms (mitochondrial and microsomal), with the same FA preference but to different degrees. Until now, only the mitochondrial isoform of GPAT (mtGPAT) has been annotated and characterized (Bionaz and Loor, 2008). The order of FA preference of mtGPAT is C16:0 > $\mathrm{C} 18: 0 \approx \mathrm{C} 14: 0>\mathrm{C} 12$; UFA are less used by mtGPAT (Monroy et al., 1973). The activity of mtGPAT is 8 to 10 times higher with C16:0-CoA than with C14:0, C18:0, or C18:1-CoA (Kinsella and Gross, 1973). Moreover, in heart and adipose tissues, GPAT are mainly mediated through the availability of FA and competing substrates (Aas and Daae, 1971). We suggest that an increase in availability of C16:0 and other long-chain SFA for lipid synthesis in mammary epithelial cells will increase the 

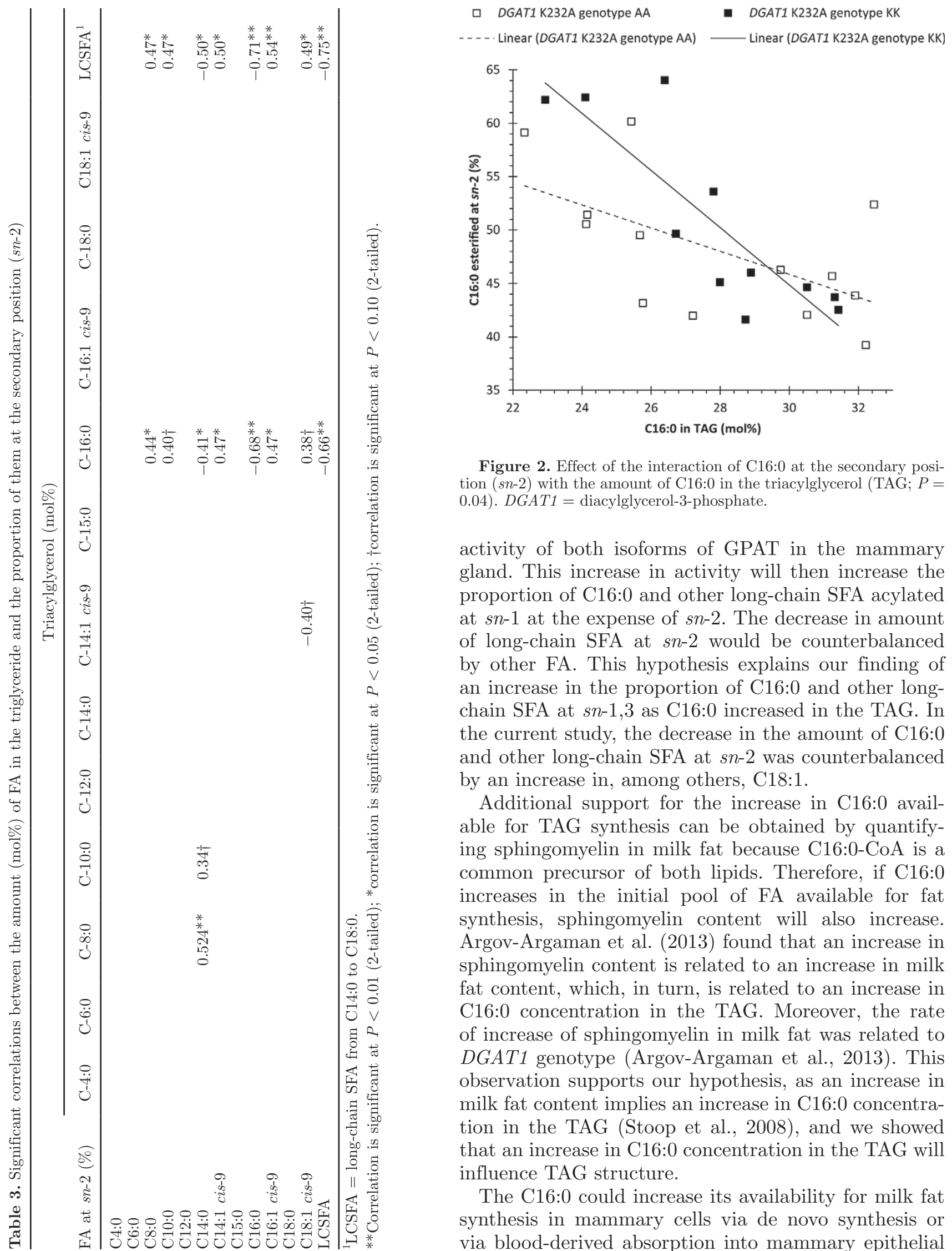

Figure 2. Effect of the interaction of $\mathrm{C} 16: 0$ at the secondary position $(s n-2)$ with the amount of C16:0 in the triacylglycerol (TAG; $P=$ 0.04). DGAT1 = diacylglycerol-3-phosphate.

activity of both isoforms of GPAT in the mammary gland. This increase in activity will then increase the proportion of C16:0 and other long-chain SFA acylated at $s n-1$ at the expense of $s n-2$. The decrease in amount of long-chain SFA at $s n-2$ would be counterbalanced by other FA. This hypothesis explains our finding of an increase in the proportion of C16:0 and other longchain SFA at $s n-1,3$ as C16:0 increased in the TAG. In the current study, the decrease in the amount of C16:0 and other long-chain SFA at sn-2 was counterbalanced by an increase in, among others, C18:1.

Additional support for the increase in C16:0 available for TAG synthesis can be obtained by quantifying sphingomyelin in milk fat because $\mathrm{C} 16: 0-\mathrm{CoA}$ is a common precursor of both lipids. Therefore, if C16:0 increases in the initial pool of FA available for fat synthesis, sphingomyelin content will also increase. Argov-Argaman et al. (2013) found that an increase in sphingomyelin content is related to an increase in milk fat content, which, in turn, is related to an increase in C16:0 concentration in the TAG. Moreover, the rate of increase of sphingomyelin in milk fat was related to DGAT1 genotype (Argov-Argaman et al., 2013). This observation supports our hypothesis, as an increase in milk fat content implies an increase in C16:0 concentration in the TAG (Stoop et al., 2008), and we showed that an increase in C16:0 concentration in the TAG will influence TAG structure.

The C16:0 could increase its availability for milk fat synthesis in mammary cells via de novo synthesis or via blood-derived absorption into mammary epithelial 
cells. De novo C16:0 is synthesized in the cytosol of the mammary epithelial cells after a series of biochemical steps that elongate $\mathrm{C} 2: 0-\mathrm{CoA}$ and $\mathrm{C} 4: 0-\mathrm{CoA}$ (Bionaz and Loor, 2008). A genetic predisposition could lead to more efficient elongation, leading to an excess of C16:0 compared with the shorter SFA, with C16:0 becoming dominant. The mechanism of stopping the elongation of $\mathrm{C} 2: 0$ and $\mathrm{C} 4: 0$ is not known but it could be linked to enzyme activity. The significant effect found in the current study for DGAT1 K232A genotype KK on the proportion of $\mathrm{C} 16: 0$ placed at $s n-2$ suggests that genetics could have an effect on TAG structure. It is plausible that DGAT1 K232A genotype KK increases the availability of C16:0 for lipid synthesis by affecting other enzymes or cell organelles related to lipid synthesis. Blood-derived C16:0 and other long-chain SFA could increase their availability for milk fat synthesis with the diet. Blood-derived FA enter the mammary epithelial cell via flip-flop and protein-mediated mechanisms (Bionaz and Loor, 2008). Once inside the cell, blood-derived and de novo-produced C16:0 long-chain SFA are activated by attaching an acyl-CoA; C16:0 and long-chain FA with CoA are transported by FABP3 to the mitochondria or endoplasmic reticulum where they serve directly as substrate for TAG synthesis (Bionaz and Loor, 2008). The increase in C16:0 and other longchain SFA available for milk fat synthesis may then result in an increased mitochondrial and microsomal GPAT activity, leading to a change in TAG structure as discussed previously. Studies on GPAT activity of mammary epithelial cells are necessary to confirm this hypothesis. Moreover, the observed effect of DGAT1 on TAG structure will need to be confirmed with a larger number of animals.

Regiospecific structural analysis of TAG with chemical or enzymatic degradation of TAG has been used to determine the average FA composition of $s n-2$ and $s n$ 1,3 of vegetable and marine oils as well as human and ruminant milk fat (Angers et al., 1998; López-López et al., 2002; Turon et al., 2002). The advantages and limitations of both approaches have been widely discussed by others (Yurkowski and Brockerhoff, 1966; Becker et al., 1993; Turon et al., 2002). Recently, new techniques to determine the regioisomerism of individual molecular species of TAG have been described (Kubo et al., 2013; Linderborg et al., 2014). However, these techniques still need to be optimized and validated to be used on bovine milk fat.

\section{Implications}

In the Netherlands, milk fat content has increased by $20 \%$ since the 1950s (Bijl et al., 2013). This increase is the result of breeding and feeding strategies in the Netherlands during the last century (Heck et al., 2009). This increase in fat content has led to higher amounts of SFA and especially of C16:0 (Stoop et al., 2008). Breeding specialists should thus be aware that the increase in SFA affects milk fat TAG structure because higher amounts of C16:0 in milk fat implies lower proportions of long-chain SFA, especially of 16:0 and C14:0, at $s n-2$ and higher proportions of C18:1 cis-9 at the same position. An increase in long-chain SFA has an effect in the thermal properties of milk fat. Because higher proportions of C16:0 at sn-2 are desired for infant nutrition (López-López et al., 2001), bovine milk fat with low concentrations of $\mathrm{C} 16: 0$ in its overall profile could better fit infant nutrition requirements. It is likely that certain milk fat fractions will give a profile richer in $\mathrm{C} 16: 0$ at $s n-2$.

\section{CONCLUSIONS}

Individual differences in milk fat TAG structures were found within a dairy cattle population. To understand the changes in TAG structure among cows, 2 approaches were used in the present study. With an intrapositional approach, the change of a FA at $s n-2$ and $s n-1,3$ was related to its total content in the TAG. With an interpositional approach, the concentration of C16:0 in the TAG was negatively correlated with the proportion of $\mathrm{C} 16: 0$, and C14:0 and was positively correlated with the proportion of $\mathrm{C} 18: 1$ cis-9 at $s n-2$. We suggest that mtGPAT indirectly controls the amount of FA that are esterified at the $s n-2$ position by selectively esterifying $\mathrm{C} 16: 0$ at $s n-1$. As a consequence, the positioning of $\mathrm{C} 14: 0$ and $\mathrm{C} 18: 1$ cis-9 at sn-2 is less dependent on its own amount in the TAG but is related to the presence of $16: 0$ at $s n-1$. The DGAT1 K232A genotype AA showed a significant effect on the proportion of C16:0 placed at sn-2; DGAT1 K232A genotype KK showed a higher change rate in the proportional positioning of C16:0 at $s n-2$. Because DGAT1 esterifies an FA at $s n-3$, we cannot draw any conclusions about the FA preference of this enzyme. Further studies could investigate the behavior of $\mathrm{C} 16: 0$ at $s n-1$ and the effect of DGAT1 polymorphism on $s n-3$ with a stereospecific analysis of milk fat TAG. High amounts of C16:0 at $s n-2$ are desirable for infant nutrition because C16:0 in human milk is mainly placed at $s n-2$. Therefore, if milk fat is used as an ingredient for infant formulas, milk fat with low amounts of C16:0 in the TAG will fit better this requirement.

\section{ACKNOWLEDGMENTS}

We thank the Consejo Nacional de Ciencia y Tecnología (CONACYT, México, Distrito Federal, Mexico) 
from the Mexican government for financial support. We thank Herman van den Brink from Qlip laboratories (Zutphen, NL) for carrying out the GC analysis, Roel van der Vaart and Alexandra Reznek from the Dairy Science and Technology Group from Wageningen University and Research Centre (WUR, Wageningen, the Netherlands) for carrying out part of the experiments, and Henk Bovenhuis (Animal Breeding and Genetics group, WUR) for his critical revision of this paper. The authors thank the Dutch Milk Genomics Initiative (Wageningen, the Netherlands) for providing the samples for this research.

\section{REFERENCES}

Aas, M., and L. N. W. Daae. 1971. Fatty acid activation and acyl transfer in organs from rats in different nutritional states. Biochim. Biophys. Acta 239:208-216.

Angers, P., É. Tousignant, A. Boudreau, and J. Arul. 1998. Regiospecific analysis of fractions of bovine milk fat triacylglycerols with the same partition number. Lipids 33:1195-1201.

Argov-Argaman, N., K. Mida, B.-C. Cohen, M. Visker, and K. Hettinga. 2013. Milk fat content and DGAT1 genotype determine lipid composition of the milk fat globule membrane. PLoS ONE 8:e68707.

Becker, C. C., A. Rosenquist, and G. Hølmer. 1993. Regiospecific analysis of triacylglycerols using allyl magnesium bromide. Lipids 28:147-149.

Bijl, E., H. J. van Valenberg, T. Huppertz, and A. C. van Hooijdonk. 2013. Protein, casein, and micellar salts in milk: Current content and historical perspectives. J. Dairy Sci. 96:5455-5464.

Bionaz, M., and J. J. Loor. 2008. Gene networks driving bovine milk fat synthesis during the lactation cycle. BMC Genomics 9:366.

Blasi, F., D. Montesano, M. De Angelis, A. Maurizi, F. Ventura, L. Cossignani, M. S. Simonetti, and P. Damiani. 2008. Results of stereospecific analysis of triacylglycerol fraction from donkey, cow, ewe, goat and buffalo milk. J. Food Compost. Anal. 21:1-7.

Bremer, J., K. S. Bjerve, B. Borrebaek, and R. Christiansen. 1976. The glycerophosphateacyltransferases and their function in the metabolism of fatty acids. Mol. Cell. Biochem. 12:113-125.

Christie, W. W. 2003. Lipid analysis: Isolation, separation, identification and structural analysis of lipids. The Oily Press Lipid Library; vol. 15. Oily Press, Bridgwater, UK.

Christie, W. W., and J. L. Clapperton. 1982. Structures of the triglycerides of cow's milk, fortified milks (including infant formulas), and human milk. J. Soc. Dairy Technol. 35:22-24.

Christie, W. W., and J. H. Moore. 1969. A semimicro method for stereospecific analysis of triglycerides. Biochim. Biophys. Acta 176:445-452.

Coleman, R. A., and D. P. Lee. 2004. Enzymes of triacylglycerol synthesis and their regulation. Prog. Lipid Res. 43:134-176.

Heck, J. M. L., H. J. F. van Valenberg, H. Bovenhuis, J. Dijkstra, and T. C. M. van Hooijdonk. 2012. Characterization of milk fatty acids based on genetic and herd parameters. J. Dairy Res. 79:39-46.

Heck, J. M. L., H. J. F. van Valenberg, J. Dijkstra, and A. C. M. van Hooijdonk. 2009. Seasonal variation in the Dutch bovine raw milk composition. J. Dairy Sci. 92:4745-4755.

ISO/IDF (International Organization for Standardization/International Dairy Federation). 2002. Milk fat-Determination of the fatty acid composition by gas-liquid chromatography. ISO 15885:2002/ IDF 184:2002. ISO, Geneva, Switzerland.

Jensen, R. G. 2002. The composition of bovine milk lipids: January 1995 to December 2000. J. Dairy Sci. 85:295-350.

Kallio, H., K. Korkiasaari, O. Sjövall, J.-P. Suomela, and K. Linderborg. 2006. The regiospecific position of $18: 1$ cis and trans monoe- noic fatty acids in milk fat triacylglycerols. J. Am. Oil Chem. Soc. 83:407-413.

Kinsella, J. E., and M. Gross. 1973. Palmitic acid and initiation of mammary glyceride synthesis via phosphatidic acid. Biochim. Biophys. Acta 316:109-113.

Kubo, A., T. Satoh, Y. Itoh, M. Hashimoto, J. Tamura, and R. B. Cody. 2013. Structural analysis of triacylglycerols by using a MALDI-TOF/TOF system with monoisotopic precursor selection. J. Am. Soc. Mass Spectrom. 24:684-689.

Linderborg, K. M., M. Kalpio, J. Mäkelä, H. Niinikoski, H. P. Kallio, and H. Lagström. 2014. Tandem mass spectrometric analysis of human milk triacylglycerols from normal weight and overweight mothers on different diets. Food Chem. 146:583-590.

López-López, A., A. I. Castellote-Bargalló, C. Campoy-Folgoso, M. Rivero-Urgel, R. Tormo-Carnicé, D. Infante-Pina, and M. C. López-Sabater. 2001. The influence of dietary palmitic acid triacylglyceride position on the fatty acid, calcium and magnesium contents of at term newborn faeces. Early Hum. Dev. 65(Suppl. 2):S83-S94.

López-López, A., M. C. López-Sabater, C. Campoy-Folgoso, M. Rivero-Urgell, and A. L. Castellote-Bargalló. 2002. Fatty acid and sn-2 fatty acid composition in human milk from Granada (Spain) and in infant formulas. Eur. J. Clin. Nutr. 56:1242-1254.

Mattson, F. H., G. A. Nolen, and M. R. Webb. 1979. Absorbability by rats of various triglycerides of stearic and oleic acid and the effect of dietary calcium and magnesium. J. Nutr. 109:1682-1687.

Monroy, G., H. C. Kelker, and M. E. Pullman. 1973. Partial-purification and properties of an acyl coenzyme A:sn-glycerol 3-phosphate acyltransferase from rat liver mitochondria. J. Biol. Chem. 248:2845-2852.

Morrison, I. M., and J. C. Hawke. 1977. Positional distribution of fatty acids in triglycerides of bovine milk fat with elevated levels of linoleic acid. Lipids 12:1005-1011.

Palmquist, D. L. 2006. Milk fat: Origin of fatty acids and influence of nutritional factors thereon. Pages 43-92 in Advanced Dairy Chemistry: Volume 2: Lipids. 3rd ed. P. F. Fox and P. L. H. McSweeney, ed. Springer, New York, NY.

Palmquist, D. L., A. D. Beaulieu, and D. M. Barbano. 1993. Feed and animal factors influencing milk fat composition. J. Dairy Sci. $76: 1753-1771$.

Parodi, P. W. 1979. Stereospecific distribution of fatty acids in bovine milk fat triglycerides. J. Dairy Res. 46:75-81.

Parodi, P. W. 1983. Relationship between fatty acid composition and triglyceride structure of bovine milk fat. J. Dairy Res. 50:443-447.

Schennink, A., W. M. Stoop, M. H. P. W. Visker, J. M. L. Heck, H. Bovenhuis, J. J. van der Poel, H. J. F. van Valenberg, and J. A. M. van Arendonk. 2007. DGAT1 underlies large genetic variation in milk-fat composition of dairy cows. Anim. Genet. 38:467-473.

Stoop, W. M., J. A. M. van Arendonk, J. M. L. Heck, H. J. F. van Valenberg, and H. Bovenhuis. 2008. Genetic parameters for major milk fatty acids and milk production traits of Dutch HolsteinFriesians. J. Dairy Sci. 91:385-394.

Tomarelli, R. M., B. J. Meyer, J. R. Weaber, and F. W. Bernhart. 1968. Effect of positional distribution on absorption of fatty acids of human milk and infant formulas. J. Nutr. 95:583-590.

Turon, F., P. Bachain, Y. Caro, M. Pina, and J. Graille. 2002. A direct method for regiospecific analysis of TAG using $\alpha$-MAG. Lipids $37: 817-821$.

Turon, F., F. Bonnot, Y. Caro, M. Pina, and J. Graille. 2003. Acyl migration incidence on accuracy of a triacylglycerol regioanalysis-A theoretical evaluation. Chem. Phys. Lipids 125:41-48.

van Valenberg, H. J. F., K. A. Hettinga, J. Dijkstra, H. Bovenhuis, and E. J. M. Feskens. 2013. Concentrations of n- 3 and n- 6 fatty acids in Dutch bovine milk fat and their contribution to human dietary intake. J. Dairy Sci. 96:4173-4181.

Yurkowski, M., and H. Brockerhoff. 1966. Fatty acid distribution of triglycerides determined by deacylation with methyl magnesium bromide. Biochim. Biophys. Acta 125:55-59. 